\title{
INTERSUBJETIVIDAD E INDIVIDUACIÓN EN HUSSERL Y HABERMAS: DE LA NECESIDAD DE UN CAMBIO DE PARADIGMA A LA COMPLEMENTACIÓN
}

\author{
INTERSUBJECTIVITY AND INDIVIDUATION IN HUSSERL AND HABERMAS: \\ OF THE NEED OF A PARADIGM SHIFT TO COMPLEMENTATION
}

\author{
Sebastián Mendl* \\ Universidad de Buenos Aires \\ Buenos Aires-Argentina \\ Recibido julio de 2019/Received July, 2019 \\ Aceptado junio de 2020/Accepted June, 2020
}

\begin{abstract}
RESUMEN
El presente trabajo reconstruye, desde una perspectiva crítica, las objeciones de Habermas desarrolladas en la Teoría de la Acción Comunicativa y sus escritos complementarios, a la teoría de la intersubjetividad husserliana. Para el autor, la construcción de una teoría de la sociedad exige un cambio de paradigma desde la filosofía de la conciencia a la filosofía del lenguaje, con el consecuente abandono de las referencias a todo punto de partida solipsista. Precisamente, desde la perspectiva habermasiana, el problema del origen de la autoconciencia encuentra su solución al referirse a la actividad social en la que el sujeto está implicado. La individuación del sujeto se constituye en el nivel de la intersubjetividad lingüística: el contexto de interacción precede a la aparición de perspectivas individuales respecto de las experiencias. Ahora bien, la presente investigación devela que la teoría de Habermas falla en demostrar que la autopercatación del sujeto es generada enteramente por las relaciones sociales. En efecto, no es posible eludir el sentido primario de la identidad que Husserl ha designado como el polo de acción y afección. Las aporías de la teoría habermasiana muestran la necesidad de reestablecer un diálogo con la fenomenología trascendental, que no parta de un cambio de paradigma, un abandono de la filosofía de la conciencia, sino de la posibilidad de un enriquecimiento recíproco entre ambas propuestas teóricas. En conclusión, una filosofía del sujeto constituye una condición necesaria para elucidar la intersubjetividad y la institución del mundo de la vida social.
\end{abstract}

Palabras Clave: Intersubjetividad, Fenomenología Trascendental, Teoría de la acción comunicativa, Individuación, Edmund Husserl.

\section{ABSTRACT}

This article reconstructs, from a critical perspective, Habermas' objections developed in the Theory of Communicative Action and its complementary writings, to Husserlian theory of intersubjectivity. For the author, the construction of a theory of society requires a paradigm shift from the philosophy of consciousness to the philosophy of language, with the subsequent abandonment of references to all solipsist starting points. Precisely, from the Habermasian perspective, the problem of the origin of self-consciousness finds its solution by referring to the social activity in which the subject is involved. The individuation of the subject is formed at the level of linguistic intersubjectivity: the context of interaction precedes the emergence of individual perspectives about experiences. The current investigation exposes that Habermas' theory fails to show that self-perception of the subject is entirely generated by social relations. In fact, it is not possible to avoid the primary sense of identity that Husserl has designated as the pole of action and affection. The aporias of the Habermasian theory show the need to reestablish a dialogue with transcendental phenomenology, which does not start from a paradigm shift, an abandonment of the philosophy of consciousness, but from the possibility of a mutual enrichment between both theoretical proposals. In conclusion, a philosophy of the subject constitutes a necessary condition to elucidate the intersubjectivity and the institution of the social life-world. Husserl.

Key Words: Intersubjectivity, Transcendental Phenomenology, Theory of communicative action, Individuation, Edmund 
LA CRÍTICA HABERMASIANA A LA FENOMENOLOGÍA TRASCENDENTAL DE EDMUND HUSSERL

Para Habermas, todo programa teórico que entienda la sociedad como un proceso generativo de una realidad estructurada en términos de sentido debe dar cuenta de quién es el sujeto de ese proceso de generación, o si es que no se da ningún sujeto (Habermas, 1989). Dentro de este marco, el autor realiza una crítica a la fenomenología husserliana por pretender derivar el mundo social y la intersubjetividad constituyente del mismo de las operaciones de un sujeto solipsista ${ }^{1}$ (Habermas, 1989).

Efectivamente, para Edmund Husserl (1986; 1976), el punto de partida para todo filosofar radical debe ser la experiencia de la propia mónada. Por ello, la fenomenología trascendental comienza instaurando un solipsismo metodológico, prescinde de todo aquello cuyo sentido se encuentre codeterminado por el otro (Husserl, 1986). La subjetividad primordial que así se consigue comprehende: 1) El cuerpo propio ${ }^{2}$; 2) un mundo primordial, la mera naturaleza a la que se puede acceder inmediatamente por medio de los sentidos (Husserl, 1986). A partir de este necesario punto de partida egológico, Husserl (1996) procura mostrar que las operaciones constituyentes no se reducen al sujeto solitario, sino que comprehenden a las ejecuciones de otras subjetividades trascendentales. Por ello, el autor, indaga la experiencia empática, en la que el Ego constituye el sentido alter ego e instituye un mundo intersubjetivo (Husserl, 1986).

Para Husserl (1986), en el campo perceptivo del mundo primordial se presentan cuerpos materiales que exhiben una semejanza con el cuerpo propio [Leib]: sus gestos, movimientos, comportamientos se asemejan a aquellos de la mónada. En virtud de esta semejanza se produce una apercepción ${ }^{3}$ analogizante [analogische Apperzeption], primer estadío de la experiencia empática. Se trata de una síntesis pasiva de parificación [Paruung], mediante la cual se transfiere al cuerpo físico que se presenta en el campo perceptivo, el sentido cuerpo propio, originariamente instituido para la propia corporalidad (Husserl, 1986; Cfr. Walton, 2001).

Se establece así una equivalencia entre los movimientos y gestos observados exteriormente y los del cuerpo propio otorgando a los primeros el sentido de los segundos (Husserl, 1986). Precisamente, la percepción de un cuerpo propio que resulta de la transferencia analogizante implica la apresentación
[Appräsentation] de un Yo, una interioridad, que gobierna ese cuerpo, está orientado por medio de su corporalidad a un mundo, tiene su propia vida de conciencia, y cuenta con una experiencia original en la que el mismo aprehende su mundo, su cuerpo y sus vivencias (Walton, 2015).

No obstante, lo esencialmente propio del otro, su flujo de vivencias, no es accesible de un modo directo, sino que exige una "cierta mediatidad de la intencionalidad" [Mittelbarkeit der Intentionalität] (Husserl, 1986, p. 175). Por ello, en un segundo momento, sobre la pasividad que instala la parificación [Paruung], es necesario un acto que verifique, de algún modo, el sentido transferido (Husserl, 1986). Para el autor (Husserl, 1986), junto con la transferencia del sentido cuerpo propio, se establece la posibilidad de esperar que ese cuerpo se comporte del mismo modo que el propio Ego en circunstancias similares. De este modo, si el hombre ve que el alter ego se enfrenta con obstáculos al caminar, supone que se desviará de ellos, del mismo modo que él lo haría en circunstancias análogas. Así, sin llegar a una presentación del curso de vivencias ajenas, en virtud de la confirmación de las esperas anticipatorias, se produce "una especie de accesibilidad verificable de lo que es originalmente inaccesible" (Husserl, 1986, p. 182). (Cfr. Walton, 2015).

Hasta este momento, se debe hablar de una empatía impropia, que equivale a la presentación del cuerpo propio extraño junto con la apresentación [Appräsentation] de un Yo que gobierna ese cuerpo (Walton, 2001). En un tercer estadío, la empatía auténtica implica la plenificación de la apresentación [Appräsentation] de una interioridad extraña por medio de una presentificación (Walton, 2001). La verdadera experiencia del alter ego se establece mediante la fantasía o imaginación, que permite al ego colocarse en el lugar del otro, como si él estuviese allí (Husserl, 1986). Así puede el sujeto imaginarse cómo el otro constituye el mundo colocándose en su lugar y presentificándose los modos de aparición de las cosas y el mundo, desde ese allí que el otro ocupa (Husserl, 1986). Ahora bien, "a partir de ahí (...) todo objeto natural experimentado y experimentable por mí en el estrato inferior [en el mundo primordial], recibe un estrato apresentativo (...), un estrato unido en una síntesis de identidad con el estrato que me es dado en primordial originalidad: el mismo objeto natural en los posibles modos de darse del otro" (Husserl, 1986, p. 194). De este modo, los entes son experimentados como 
objetos aprehendidos también por otros, y, por ello, trascendentes a la propia mónada. La empatía habilita así la constitución de una naturaleza intersubjetiva, que constituye la condición de posibilidad de las formaciones de sentido común (Husserl, 1986).

Asimismo, junto con la constitución del sentido alter ego en la experiencia empática, se devela el recíproco existir el uno para el otro (Husserl, 1986). Ahora, el hombre se comprende como alter ego para el Otro, como siendo constituido de modo análogo a como el otro es constituido por él (Husserl, 1986). Así como el cuerpo orgánico ajeno se encuentra en el campo de percepción del ego, también su propio cuerpo orgánico se encuentra en el campo perceptivo del alter ego. De este modo, la empatía constituye la condición de posibilidad de toda comunicación e interacción recíproca entre mónadas (Husserl, 1986). Efectivamente, sobre esta experiencia se erigen los actos sociales, es decir, aquellos actos guiados por una voluntad de comunicación (Husserl, 1986).

Habermas (1989), refiriéndose exclusivamente a la presente propuesta, desarrollada por Husserl en las Meditaciones Cartesianas (1986), destaca dos problemáticas fundamentales de la fenomenología de la intersubjetividad, ambas producto del punto de partida solipsista de la presente teoría: (i) Husserl es incapaz de demostrar cómo el ego experimenta a otro Yo, como un sujeto constituyente y no un mero objeto constituido; (ii) en segundo lugar, la perspectiva fenomenológica no logra elucidar la constitución de un mundo en común.

(i) En primer lugar, el autor (1989) destaca que Husserl es incapaz de explicar por qué en el mundo primordial, en el que solo un cuerpo destaca como la propia corporalidad originariamente vivida, habría de poderse seleccionar de entre todos los cuerpos un subconjunto de los mismos como potenciales corporalidades de otros sujetos. En efecto, la corporalidad vivida, así como es analizada por Husserl, es radicalmente distinta al cuerpo visto desde afuera (Habermas, 1989; Cfr. Mensch, 2010). Por ello, no es posible apoyar en este punto la transferencia analogizante de sentido, el cuerpo que se presenta en el campo primordial no posee ningún lado yoico (Habermas, 1989).

Por otra parte, para Habermas (1989), tampoco es posible justificar un acto que verifique el sentido transferido por la parificación. Precisamente, para el autor (Habermas, 1989), solo se pueden entender los movimientos de otro cuerpo como un accionar conforme a intenciones identificables, al ser referidos a reglas que fijan un sistema de símbolos y determinan qué rasgos físicos han de valer como signos, así como los qué significados pueden atribuirse a los mismos en diversas situaciones. Ahora bien, las reglas, a diferencia de las meras regularidades empíricas, solo existen como tal a partir de su validez intersubjetiva (Habermas, 1987b). En consecuencia, comprender gestos significativos presupone la habilidad de participar en una práctica pública con al menos otro sujeto (Habermas, 1989). De este modo, la elucidación husserliana de un alter ego presupone la intersubjetividad que se intenta elucidar (Habermas, 1989).

En síntesis, el punto de partida solipsista de la presente teoría inhabilita la elucidación de otras subjetividades constituyentes (Habermas 1989). Por una parte, en el campo primordial no se presenta ningún cuerpo análogo a la propia corporalidad y, en segundo lugar, los comportamientos ajenos a lo sumo se podrían comprender como regularidades empíricas, que valen para todo objeto del entorno inmediato.

(ii) El segundo problema destacado por Habermas (1989), también es producto del punto de partida solipsista de la teoría fenomenológica. En tanto para Husserl el ego trascendental de quién reflexiona constituye el horizonte último de legitimación, se establece una asimetría entre el ego y el alter, quedando inhabilitada así la posibilidad de una comunicación auténtica (Habermas, 1989).

Husserl intenta establecer la reciprocidad entre subjetividades mediante la invocación de la intercambiabilidad del "aquî" de la propia experiencia corporal y el "allí̂" de la experiencia corporal ajena (Habermas, 1989). Ahora bien, incluso previo al encuentro con el alter ego, el sujeto debería ocupar virtualmente todas las posibles localizaciones (Habermas, 1989). De no ser ese el caso, el sujeto no sería capaz de presentificarse la experiencia del mundo del alter ego (Habermas, 1989). Por ello, para Habermas (1989), toda comprobación que los otros pueden suministrar a las propias percepciones no adhiere nada que la propia comprobación no podría ya proveer. Ahora bien, 
"si reconocer la existencia de otra perspectiva localizada en el mundo no agrega nada que no se encuentre disponible en principio a mi horizonte solipsístico de experiencias posibles, entonces ocupar la posición del otro mediante una presentificación no puede ser lo que desafía la primacía y la autosuficiencia de mi propia conciencia egológica del mundo, y no puede ser lo que establezca al alter ego como genuinamente otro"4 (Russel, 2011, p. 54).

Por este motivo, permanece imposibilitada la constitución de un mundo común a ambos sujetos, una naturaleza intersubjetiva, trascendente a la experiencia de la propia mónada (Russel, 2011). Todas las perspectivas espaciales de los alter egos son asimiladas a la modalidad de perspectivas espaciales que el ego original ya anticipa por adelantado y ocupa virtualmente (Habermas, 1989). Habermas (1989) concluye que el modelo de Husserl no puede ofrecer ninguna forma de explicar la generación de experiencia intersubjetiva.

En conclusión, para Habermas (1989), el punto partida solipsista de la fenomenología trascendental, inhabilita la comprensión de la intersubjetividad y de la constitución de un mundo-de-la-vida social. Por ello, para el autor, la construcción de una teoría de la sociedad exige un cambio de paradigma desde la filosofía de la conciencia a la filosofía del lenguaje, con el consecuente abandono de las referencias a una conciencia intencional y a todo punto de partida monadológico (Habermas, 1989).

\section{UNA TEORÍA DE LA SOCIEDAD PLANTEADA EN TÉRMINOS DE COMUNICACIÓN}

La propuesta de Habermas desarrollada en

la Teoría de la Acción Comunicativa entiende el despliegue de la vida social como un proceso de generación mediado por actos del habla: la realidad social descansa en la facticidad de las pretensiones de validez implicadas en productos simbólicos (Russel, 2017). Precisamente, siguiendo a Wittgenstein, para Habermas (1989) el sentido debe ser interpretado por referencia a los juegos del lenguaje puestos en juego en la comunicación y el discurso.

Por ello, Habermas (1989), partiendo de la filosofía del lenguaje, establece una recepción crítica del concepto husserliano 'mundo-de-la-vida'5 . El autor (1989) propone dos movimientos esenciales para volver fructífero el análisis husserliano del mundo-de-la-vida social: (i) la redescripción de las estructuras de sentido del mundo de la vida en términos de pretensiones de validez; (ii) el análisis de estas pretensiones de validez en términos de estructuras del lenguaje transindividuales y el uso pragmático del mismo.

En palabras de Habermas (1987b),

\begin{abstract}
"Si ahora abandonamos las categorías de la filosofía de la conciencia en que Husserl trata la problemática del mundo de la vida, podemos representarnos este como un acervo de patrones de interpretación transmitidos culturalmente y organizados lingüísticamente (...). Este acervo de saber provee a los participantes en la comunicación de convicciones de fondo aproblemáticas (...) y de esas convicciones de fondo se forma en cada caso el contexto de los procesos de entendimiento, en los que los participantes hacen uso de definiciones acreditadas de la situación o negocian definiciones nuevas" (p. 176-178).
\end{abstract}

Para Habermas (1987b), el mundo en común no es producto de la habilidad del ego de ocupar en la imaginación el lugar espaciotemporal ajeno -es decir, la acumulación de perspectivas espaciales-, sino de la capacidad de entender las pretensiones de validez erigidas por otros en las acciones comunicativas. Por su parte, la subjetividad se comprende ahora como enraizada en el mundo a la vez que constituyente del mismo en virtud de su capacidad de utilizar el lenguaje para entenderse con los otros acerca de algo sobre el trasfondo intersubjetivamente compartido que es el mundo de la vida ${ }^{6}$ (Duarri Cánovas, 1994).

Ahora bien, la capacidad lingüística constituye un logro tardío en el desarrollo del infante. Por ello, siguiendo a Mead, Habermas (1987b) analiza cómo el habla proposicionalmente diferenciada emerge de la capacidad comunicativa mediante gestos significativos, común a varias especies animales.

El gesto, primer acto significativo, adquiere su significado prelingüísticamente mediante un acto perceptivo complejo en donde un estímulo es asociado con una respuesta (1987b). En palabras del autor:

"En el lenguaje de ademanes, las relaciones que se dan entre el gesto del primer organismo y la acción subsiguiente a ese gesto, de un lado, y la reacción comportamental de un segundo organismo estimulada por ese gesto, de otro, constituyen, pues, el fundamento 
objetivo del significado que el gesto de uno de los participantes en la interacción cobra en cada caso para el otro. Y como el gesto del primer organismo viene materializado por los elementos iniciales de una reacción motriz que se presenta reiteradamente y que en este sentido constituye una indicación del estado que resultará del movimiento completo, el segundo organismo puede reaccionar a él como si fuera expresión de la intención de producir ese resultado. Con lo cual da al gesto un significado que, por de pronto, solo puede tenerlo para él" (Habermas, 1987b, p. 18).

Posteriormente, al reaccionar el segundo organismo a los ademanes del primero con una determinada conducta, y al reaccionar, a su vez, el primer organismo a los elementos iniciales de esa reacción comportamental del segundo, ambos expresan cómo interpretan, es decir, cómo entienden los gestos del otro (Habermas, 1987b).

Precisamente, deben darse tres transformaciones para que la presente interacción mediado por gestos se convierta en una interacción simbólica (Habermas, 1987b): (i) el gesto significativo debe transformarse en símbolos mediante sustitución de los significados que solo valen para cada uno de los organismos, por significados que sean idénticos para todos los participantes; (ii) la relación causal estimulo-reacción-estímulo precisa ser reemplazada por la relación interpersonal entre hablante y destinatario; (iii) tiene que producirse un cambio estructural de la interacción, de modo que los participantes distingan entre actos de entendimiento y acciones orientadas al éxito.

Por último, el autor distingue la etapa de la interacción simbólicamente mediada y la etapa de la comunicación dirigida por normas (Habermas, 1987b). El presente estadío representa la constitución de un comportamiento regido por reglas -las que solo existen como tal a partir de su validez intersubjetiva-, un comportamiento que puede ser explicado en términos de una orientación por convenciones semánticas (Habermas, 1987b). Se establece ahora una reestructuración de los motivos de la acción, se trata de un "tránsito desde un modo de control de la interacción prelingüístico y ligado a los instintos a un modo del control dependiente del lenguaje y ligado a una tradición cultural" (Habermas, 1987b, p. 42).

Es preciso destacar que el autor busca sortear en el presente relato de la génesis de la capacidad lingüística y del comportamiento regido por reglas la presuposición de un campo de experiencia primordial, a partir de ello elucidar la existencia del alter ego. No obstante, en la teoría habermasiana (1987b), la subjetividad no es eliminada. Pero ahora la individuación del sujeto se comprende como formada en el nivel de la intersubjetividad lingüística: "Es, pues, manifiesto que también la individualidad es un fenómeno generado socialmente, el cual es resultado del proceso mismo la socialización y no expresión de unas pulsiones residuales que escapen a la socialización" (Habermas, 1987b, p. 86).

Precisamente, para Habermas, el hablante, al plantear en su acto de habla una pretensión de validez susceptible de crítica a un oyente, se presenta como un sujeto capaz de dar cuenta de los motivos "subjetivos" de su accionar (Habermas, 1987b). La estructura de la intersubjetividad lingüística, que fija los roles comunicativos de hablante e interpelado, obliga a los participantes a actuar bajo la presuposición de que cada uno de ellos es capaz de responder de sus actos (Habermas, 1987b).

Por ello, Habermas identifica el principio de individuación con la estructura de perspectivas establecidas en los roles comunicativos de la primer, segunda y tercera persona (Habermas, 1987b). En la presente situación se encuentra el sentido de la expresión "yo", la que establece una pretensión de validez peculiar, la de la autenticidad (Habermas, 1987b). En palabras del autor:

"La expresión yo (...) indica la actitud
pragmática o la perspectiva en la que o desde
la que el hablante se manifiesta (...) Con la
perspectiva de primera persona adopta el
papel consistente en presentarse a sí mismo,
de modo que le pueden ser atribuidos los
deseos, sentimientos, intenciones, opiniones,
etc., que expresa" (Habermas, 1987b, p. 150).

En conclusión, para Habermas, el niño desarrolla un Self en la medida en que se forma para él un mundo social al que él pertenece y, simultáneamente, un mundo subjetivo deslindado del mundo externo de los acontecimientos y de las normas, y al que él tiene un acceso privilegiado (Habermas, 1987b):

"El muchacho a medida que se apropia cognitivamente el mundo social de relaciones interpersonales legítimamente reguladas, a medida que desarrolla el correspondiente 
sistema de controles internos y aprende a orientar su acción por pretensiones normativas de validez, traza unos límites cada vez más claros entre un mundo externo condensado en realidad institucional y el mundo interno de las licencias espontáneas que no pueden salir al exterior a través de acciones conformes a las normas, sino solo a través de la autopresentación comunicativa" (Habermas, 1987b, p. 64).

Así, siguiendo a Mead, para Habermas, la solución al problema psicológico esencial de la autoconciencia se encuentra al referirse al proceso de conducta o actividad social en el que está implicada la persona (Dews, 1995). El contexto de interacción precede a la aparición de perspectivas individuales acerca de esa interacción (Dews, 1995).

\section{LA FENOMENOLOGÍA TRASCENDENTAL Y EL GIRO LINGÜÍSTICO: DEL CAMBIO DE PARADIGMA A LA COMPLEMENTACIÓN}

En consonancia con los análisis habermasianos, Husserl expresa en sus manuscritos póstumos -no analizados por Habermas-, que la constitución de un mundo común se produce, principalmente, mediante los actos lingüísticos guiados por una voluntad de comunicación: "Yo veo, oigo y experimento no solo con mis sentidos, sino también con los de los Otros y el Otro experimenta no solo con los suyos, sino también con los míos; ello ocurre gracias a la transmisión de conocimientos" (Husserl, 1987, p. 150). Para el autor, mediante eventuales confirmaciones y correcciones habilitadas por la comunicación, se establece un mundo compartido por los participantes (Husserl, 1976). Por ello, Husserl expresa que "el mundo-de-la-vida -el mundo para todos nosotros- es idéntico con el mundo del que podemos discutir comunitariamente" (Husserl, 1976, p. 213).

Además, en dichos textos, Husserl (1987) también postula que es preciso un reconocimiento intersubjetivo, en la formación de la autoconciencia personal. No obstante, mientras que Habermas (1987b) afirma que no hay ninguna subjetividad prelingüística que preceda la relación a uno mismo que es puesta en y mediante la estructura lingüística de la intersubjetividad, Husserl (1987), distingue diferentes niveles de estructura yoica. Establece una diferencia entre el yo-primordial, el Yo como unidad en el discurrir de las vivencias, como polo de acción y afección, y el yo personal, en toda su concreción, constituido mediante el encuentro con el alter ego (Husserl, 1987). En palabras del autor:

\begin{abstract}
"El polo de todas las afecciones y acciones (...), el sujeto que atraviesa la corriente de vivencias y es, como tal, el sujeto permanente de un aspirar en múltiples modalidades, se convierte en un yo, y con ello en un sujeto personal, gana una autoconciencia personal, en la relación Yo-Tú, en la comunidad de aspiraciones y voluntades que la comunicación hace posible" (1987, p. 138).
\end{abstract}

Ahora bien, la presente estratificación de la estructura yoica permite responder a algunos interrogantes que quedan en suspenso en la Teoría de la Acción Comunicativa habermasiana. Investigaciones previas (Russel, 2011; 2017) han destacado que el análisis habermasiano de la génesis de los gestos significativos, primer paso en la constitución de la comunicación lingüística, devela un conjunto de condiciones de posibilidad subjetivas.

En primer lugar, la captación de un gesto significativo constituye un acto complejo que implica fases temporales y la asociación de las mismas (Russel, 2011). Asimismo, los objetos complejos de la percepción deben ser asociados con una respuesta en la propia subjetividad (Russel, 2011). En síntesis, incluso los gestos significativos implican la propia capacidad de experimentar al otro como un objeto temporal complejo distinto de uno y parificar su respuesta con la acción propia.

Ahora bien, la capacidad de identificar la reacción del otro como dirigida hacia uno ya presupone una conciencia de sí mismo, es necesario que el implicado pueda comprenderse como objeto para sí (Russel, 2011). De lo contrario, no se podría distinguir estas reacciones de aquellas dirigidas hacia terceros (Dews, 1995). De este modo, el inicio del relato de Mead, retomado por Habermas, presupone un sentido de identidad primario (Dews, 1995). Del mismo modo, la intersubjetividad lingüística no puede ser el fundamento de la individuación: el hablante debe ser consciente de que la pretensión de validez de autenticidad es sobre sí mismo, y este núcleo cognitivo aún requiere una explicación en la obra habermasiana (Dews, 1995). En conclusión, el relato de Habermas falla en demostrar que la autopercatación del sujeto es generada enteramente por las interacciones comunicativas. 
En efecto, no es posible eludir el sentido primario de la identidad que Husserl ha designado como el polo de acción y afección, en búsqueda de destacar dentro de la mónada un momento de centralización y unidad por el que "el ego mismo es existente para sí mismo con una continua evidencia" (1986, p. 118). Para el autor (1986), un sentido mínimo de "propiedad" caracteriza la experiencia de todo sujeto, siendo una condición de posibilidad de la posterior reflexión acerca del acto y del establecimiento de relaciones intersubjetivas. Las experiencias no ocurren "en el sujeto" sino que también son dadas para él y, por ello, experimentadas como propias (Husserl, 1997). No hay primero un mero acontecer de la experiencia al que sigue luego algún modo de adscripción a alguien (Husserl, 1997). Por el contrario, el ego se percata de sí mismo de un modo radical y peculiar invariablemente involucrado en toda experiencia (Husserl, 1997).

En este sentido, la psicología científica muestra que ya los infantes de 24 horas pueden discriminar entre el estímulo táctil externo y la autoestimulación (Rochat, 2001). Asimismo, ya en las primeras semanas de vida los niños son capaces de detectar qué objetos se encuentran a su alcance (Neisser, 1993). La percepción de objetos involucra información de la propia subjetividad, en tanto el infante debe estar al tanto de la posición del objeto en relación con él (Neisser, 1993). A base de estos estudios, Gallagher y Zahavi (2013) argumentan, desde una perspectiva fenomenológica, que el infante desarrolla un temprano sentido del Ego, basado en la experiencia sensorial.

Ahora bien, las experiencias no transcurren sin dejar rastros (Husserl 1997). Cuando la experiencia presente ya no se vive "en su actualidad", se establece una sedimentación de la misma, sobre la que se posteriormente se erigen las futuras experiencias (Husserl 1997). El Ego, como polo de acción y afección, es primariamente un sustrato de habitualidades (Husserl, 1987). Por ello, junto con el sentido primario de propiedad que caracteriza toda experiencia, para Husserl, "tiene sentido hablar de la individualidad como estilo total y hábito del sujeto, que atraviesa, como una unidad concordante, por todas las maneras de comportamiento, por todas las actividades y pasividades" (Husserl, 1997, p. 325). En efecto, en los primeros meses de vida, los infantes "se experiencian a sí mismos, experiencian lo que son, cómo se mueven, lo que están haciendo y si una cierta acción es la suya propia o no" (Gallagher y Zahavi, 2013, p. 308).

En conclusión, el sentido primario de la identidad husserliano permite dar cuenta de la individuación primordial que se encuentra supuesto en la lectura habermasiana de Mead y en el análisis pragmático de las pretensiones de validez de autenticidad. El análisis de la intersubjetividad de Habermas no puede ser el suelo fundante de la subjetividad. Por ello, una teoría de la sociedad y de la comunicación que fundamente el desarrollo de las ciencias sociales y permita una auténtica crítica al mundo contemporáneo precisa un análisis tanto del principio de individuación como de la capacidad de experimentar objetos temporales complejos: problemáticas que para Husserl (1973c) se encuentran íntimamente relacionadas y constituyen el nivel inferior del examen fenomenológico.

Las dificultades aquí señaladas en la teoría habermasiana muestran la necesidad de reestablecer un diálogo que no parta de un cambio de paradigma, un abandono de la filosofía de la conciencia, sino de un enriquecimiento recíproco entre ambas propuestas. En este sentido, Zahavi (2001) destaca que lo que está en juego entre la propuesta habermasiana y la fenomenología de Edmund Husserl es más una diferencia de intereses que una confrontación radical. Pese a sus diferenciaciones en los varios conceptos de identidad, Habermas permanece exclusivamente interesado en explorar la identidad personal en término de socialización (Zahavi, 2001). En contraste, no aborda en su obra el problema de la individuación del flujo de conciencia en la multiplicidad de experiencias, y sus pocas referencias a este problema indican que la entiende exclusivamente como una diferencia física que está asegurada por la contingencia del sustrato orgánico (Zahavi, 2001). No obstante, reducir el concepto primario de identidad a una mera naturaleza orgánica, implicaría recaer en el objetivismo naturalista que tanto Habermas como Husserl intentaban eludir (Zahavi, 2001).

Precisamente, una filosofía del sujeto no es una barrera para una teoría en términos de comunicación como la habermasiana. Por el contrario, hablar de intersubjetividad presupone la autonomía y la diferencia entre subjetividades (Habermas, 1989). El ego y el alter ego solo pueden mantener una relación intersubjetiva mientras se reconozcan el uno al otro en su identidad y diferencia (Zahavi, 2001). Toda relación concreta entre sujetos presupone 
la previa pluralidad o diferencia entre corrientes de conciencia (Zahavi, 2001). Por ello, eliminar la diferencia entre sujetos es eliminar la comunicación (Zahavi, 2001).

De este modo, incluso si las críticas habermasianas muestran las dificultades del intento husserliano desarrollado en las meditaciones cartesianas y de abordar la intersubjetividad, las investigaciones precedentes develan la necesidad de una complementación entre un análisis fenomenológico de la conciencia y la teoría de la comunicación. Más aún, si bien un examen completo de la intersubjetividad husserliana y sus aportes a la totalidad de la teoría habermasiana excede los límites de este trabajo, es preciso destacar que en sus manuscritos el creador de la fenomenología vuelve complejo y amplía su examen de la intersubjetividad ${ }^{7}$.

Para Husserl, la transferencia analogizante de sentido y la experiencia empática, solo son posibles porque el infante ya se encuentra vinculado a los otros por medio de formas pasivas, instintivas, afectivas y corporales de intersubjetividad (Husserl, 1973c). Husserl (1973c) enfatiza, como inherente a la especie humana, un instinto de comunicación que establece una intersubjetividad primaria entre el infante y sus cuidadores. Para el autor, el infante, previo a cualquier juicio activo, se encuentra en búsqueda de afecto y contacto, una tendencia que solo puede cumplirse con otra subjetividad.

Precisamente, en una protoempatía, se establece un vínculo primario, una unificación del operar propio y ajeno en un ámbito puramente pasivo e inmediato (Husserl, 1973c; Cfr. Rodemeyer, 2006). En la protoempatía el infante descubre la continuidad, pero no la unidad, de su propio flujo de conciencia con el del alter ego y constituye un mundo común a ambos (Husserl, 1973c; Cfr. Walton 2015).

En este sentido, investigaciones en cognición social han analizado el establecimiento de una socialidad primaria en los infantes, una forma de interacción primitiva basada en patrones rítmicos y la estructura melódica de los actos (Stern, 1985; Trevarthen 1979; Trevarthen and Reddy 2006). El comportamiento motor y vocal de los infantes se encuentra sincronizado temporalmente con el rendimiento corporal de sus cuidadores (Stern, 1985; Trevarthen 1979). Por ejemplo, el ritmo vocal de las madres se correlacionó positivamente con el compás de movimientos realizado por el infante (Stern, 1985). La estructura rítmica y temporal del comportamiento que expresa estados afectivos juega un papel primordial en las relaciones primarias entre el infante y sus cuidadores (Pokropski, 2015). La estructura diádica, basada en patrones temporales, que emerge entre el Self y el otro, constituye una "resonancia intercorporal" (Froese y Fuchs 2012): las emociones físicamente expresadas de un sujeto afectan la experiencia corporal del otro y viceversa. Este vínculo pasivo incluso afecta el movimiento básico de la vida. Spitz (1945) descubrió que los bebés institucionalizados que se ven privados de cualquier relación de apego caen en una profunda apatía y depresión, incluso hasta el punto de morir por infecciones menores.

Ahora bien, el establecimiento de la presente intersubjetividad primaria en la protoempatía implica la capacidad de experimentar objetos temporales complejos, así como habilidades kinestésicas. Por ello, Husserl no renuncia al ego primordial, que constituye la condición de posibilidad del presente vínculo intersubjetivo. En efecto, como se ha desarrollado previamente, las experiencias previas al establecimiento de la protoempatía se caracterizan por un sentido mínimo de propiedad, y mediante la sedimentación de los actos se conforma una individualidad primaria.

En conclusión, en primer término, los manuscritos de Edmund Husserl promueven la construcción de una teoría de la intersubjetividad que no implique el abandono de la filosofía del sujeto. Por otra parte, el analisis husserliano complementa el análisis de la intersubjetividad lingüística desarrollado por Habermas con un examen de la intersubjetividad primaria establecida prerreflexivamente. De este modo, los manuscritos husserlianos, al dar respuesta a algunas de las críticas habermasianas, que el mismo Husserl ha reconocido (1973c), y a las deficiencias del análisis habermasiano de la génesis de la intersubjetividad, promueven un nuevo diálogo entre ambas teorías. 


\section{BIBLIOGRAFÍA}

Dews, P. (1995). The limits of disenchantment: Essays on contemporary European Philosophy. London-New York: Verso.

Duarri Cánovas, M. (1994). Mundo de la vida y Acción comunicativa. En San Martín (Ed.) Sobre el concepto del mundo de la vida. Actas de la II Semana Española de Fenomenología (pp. 329-336). Madrid: UNED.

Fairtlough, G. H. (1991). Habermas' concept of "Lifeworld". Systems practice, 4(6), 547-563.

Froese, T., y Fuchs, T. (2012). The extended body: a case study in the neurophenomenology of social cognition. Phenomenology and the Cognitive Sciences, 11(2), 205-235. Url: https://link. springer.com/article/10.1007/s11097-012-9254-2.

Fultner, B. (2001). Intelligibility and conflict resolution in the lifeworld. Continental philosophy review, 34(4), 419-436.

Gallagher, S., \& Zahavi, D. (2013). La mente fenomenológica. Alianza Editorial.

García Marzá, M. (1994). El mundo de la vida: Husserl y Habermas. En San Martín (Ed.) Sobre el concepto del mundo de la vida. Actas de la II Semana Española de Fenomenología (pp. 241-258). Madrid: UNED.

Habermas, J. (1987a). Teoría de la acción comunicativa. Tomo I: Racionalidad de la acción y racionalización social. Madrid: Taurus.

Habermas, J. (1987b). Teoría de la acción comunicativa. Tomo II: Crítica de la razón funcionalista. Madrid: Taurus.

Habermas, J. (1989). Teoría de la acción comunicativa: complementos y estudios previos. España: Cátedra.

Husserl, E. (1973a). Zur Phänomenologie der Intersubjektivität: texte aus dem nachlass: erster teil, 1905-1920. La Haya: Martinus Nijhoff.

Husserl, E. (1973b). Zur Phänomenologie der Intersubjektivität. Texte aus dem Nachlass. ZweiterTeil: 1921-1928. La Haya: Martinus Nijhoff.

Husserl, E. (1973c). Zur Phänomenologie der Intersubjektivität. Texte aus dem Nachlass. DritterTeil: 1929-1935. La Haya: Martinus Nijhoff.

Husserl, E. (1976). Die Krisis der europäischen Wissenschaften und die transzendentale Phänomenologie. Eine Einleitung in die phänomenologische Philosophie. Husserliana VI. Den Haag: Martinus Nijhoff.

Husserl, E. (1986). Meditaciones cartesianas. Madrid: Tecnos.

Husserl, E. (1987). El espíritu común (Gemeingeist) I y II. Thémata. Revista de Filosofía, 4, 131-158.

Husserl, E. (1993). Die Krisis der europäischen Wissenschaften und die transzendentale Phänomenologie. Ergänzungsband. Texte aus dem Nachlass 1934-1937. Dordrecht: Kluwer Academic Publishers.

Husserl, E. (1997). Ideas II. Investigaciones fenomenológicas sobre la constitución. México: Universidad Nacional Autónoma de México.
Husserl, E. (2008). Die Lebenswelt. Auslegungen der vorgegebenen Welt und ihrer Konstitution. Texte aus dem Nachlass (1916-1937). Husserliana XXXIX. Dordrecht: Springer.

Mensch, J. (2010). Husserl's Account of Our Consciousness of Time. Milwaukee: Marquette University Press.

Neisser, U. (1993). The self-perceived. In U. Neisser (ed.), The Perceived Self: Ecological and Interpersonal Sources of SelfKnowledge (pp. 3-21). New York: Cambridge University Press.

Pokropski, M. (2015). Timing together, acting together. Phenomenology of intersubjective temporality and social cognition. Phenomenology and the Cognitive Sciences, 14(4), 897-909.

Rabanaque, L. R. (2013). Naturaleza, cuerpo, cultura. En Actas del XV. Congreso Nacional de Filosofía AFRA (EDUNTREF). CD-ROM. (ISBN 978-987-1889-10-5).

Rizo-Patrón, R. (2010). Diferencia y otredad desde la fenomenología de Husserl. Areté, 22(1), 87-106.

Rochat, P. (2001). The Infant's World. Cambridge, MA: Harvard University Press.

Rodemeyer, L. M. (2006). Intersubjective Temporality. It's about time. Springer, Dordrecht.

Russell, M. (2011). On Habermas's critique of Husserl. Husserl studies 27(1), 41-62. Url: https://link.springer.com/article/10.1007/ s10743-010-9080-8

Russell, M. (2017). Habermas and the 'Presupposition' of the Common Objective World. Metodo, 172-193.

Lee, N. I. (2019). The Pluralistic Concept of the Life-World and the Various Fields of the Phenomenology of the Life-World in Husserl. Husserl Studies, 1-22.

Spitz, R. A. (1945). Hospitalism: An inquiry into the genesis of psychiatric conditions in early childhood. Psychoanalytic Study of the Child, 1, 53-74.

Stern, D.N. (1985). The interpersonal world of the infant. A view from psychoanalysis and developmental psychology. London: Karnac Books.

Trevarthen, C. (1979). Communication and cooperation in early infancy: A description of primary intersubjectivity. In M. Bullow (Ed.), Before speech (pp. 15-39). Cambridge: Cambridge University Press.

Trevarthen, C., \& Reddy, V. (2006). Consciousness in infants. In M. Velman \& S. Schneider (Eds.), A companion to consciousness (pp. 58-75). Oxford: Blackwell.

Walton, R. (2001). Fenomenología de la empatía. Philosophica, $24,409-428$.

Walton, R. J. (2015). Intencionalidad y horizonticidad. Colombia: Editorial Aula de Humanidades.

Zahavi, D. (2001). Husserl and transcendental intersubjectivity: A response to the linguistic-pragmatic critique. Ohio: Ohio University Press. 


\section{NOTAS}

1 Habermas menciona en múltiples textos sus reparos con la fenomenología trascendental husserliana (Russel, 2010). No obstante, debido a la extensión de la obra del autor, el presente trabajo se limita al análisis de las críticas y contrapropuestas desarrolladas en la Teoría de la acción comunicativa (Habermas, 1987a; 1987b) y sus escritos complementarios (1989).

2 El propio cuerpo exhibe, en relación con cualquier otro cuerpo, una manera de darse peculiar (Husserl, 1997). Se tiene experiencia de él al mismo tiempo como una cosa "objetiva" que está en el mundo y como una cosa "subjetiva" que es relativa a la propia persona. El cuerpo como fenómeno experienciado tiene un lado corpóreo y un lado yoico (Husserl, 1997). En cuanto cosa del mundo, posee las propiedades de los entes materiales: se extiende en el tiempo, ocupa un lugar en el espacio y está sometido a la interacción con otros cuerpos físicos (Rabanaque, 2013). No obstante, difiere esencialmente de todo objeto físico, en tanto siempre es "co-percibido" en la percepción de los otros cuerpos, está localizado en una cercanía absoluta que no admite distancia y, por ello, es el punto cero de toda orientación en el mundo, tanto en el espacio como en el tiempo (Rabanaque, 2013). Se trata de un cuerpo subjetivo, "órgano" del yo, tanto en calidad de centro de afección del mundo sobre el yo, es decir, en cuanto cuerpo estesiológico o sintiente, como de centro de acción del yo sobre el mundo, en cuanto cuerpo volitivo o moviente (Rabanaque, 2013).

3 Husserl establece una distinción entre percepción, apresentación [Appräsentation] y presentificación [Vergegenwärtigung] (Walton, 2001). En primer lugar, la percepción es una presentación que da el objeto en el presente como algo que está ahí en persona o en carne y hueso. En segundo término, la apresentación [Appräsentation], que se enlaza siempre con la percepción, va más allá de ella e implica un excedente no dado. Por ejemplo, la percepción de una cara del objeto implica la apresentación del resto de los perfiles. Por último, la presentificación [Vergegenwärtigung] constituye un acto que da al objeto, pero no en persona como en la percepción: la imaginación, la fantasía, entre otros (Walton, 2001).

$4 \quad$ Todas las traducciones pertenecen al autor del presente trabajo.

$5 \quad$ Para Husserl (1976), el mundo de la vida [Lebenswelt] es el correlato intencional de la vida trascendental. No obstante, se trata de un concepto complejo, el que recibe diferentes descripciones en la obra husserliana y ha sido objeto de múltiples intentos de clasificación en la bibliografía especializada. Para un examen de la multiplicidad de sentidos del presente concepto, véase Lee (2019). Por otra parte, un análisis de las disidencias en la obra de Husserl y Habermas respecto del presente concepto puede encontrarse en García Marzá (1994).

6 La reapropiación habermasiana, realizada a partir de la filosofía del lenguaje, del concepto fenomenológico "mundo de la vida" [Lebenswelt] constituye un eje fundamental de la teoría de la acción comunicativa (Fairtlough, 1991). En efecto, el mundo de la vida, en tanto trasfondo atemático de convicciones, habilita la comunicación entre los hablantes y, simultáneamente, es el producto de dicha comunicación (García Marzá, 1994). Siguiendo a Faitlough (1991) es posible destacar cinco funciones del presente concepto en la obra habermasiana: (i) en primer lugar, permite elaborar una explicación de la constitución del mundo objetivo, el mundo social y el mundo subjetivo; (ii) en segundo término, devela el modo en que las comunidades y los individuos se desarrollan y perduran en el tiempo; (iii) por otra parte, contribuye a explorar los vínculos entre los debates racionales explícitos y las convicciones no formuladas pero compartidas por los hablantes; (iv) en cuarto lugar, permite establecer un contraste entre las acciones comunicativas y las acciones estratégicas; (v) por último, la diferencia entre sistema y mundo de la vida es utilizada por Habermas para explicar la crisis propia de las sociedades modernas.

Para una reconstrucción de las múltiples conceptualizaciones del mundo de la vida en la obra de Habermas, véase Fultner, (2001). 7 Los manuscritos husserlianos acerca de la intersubjetividad, desarrollados desde 1905 a 1935, se han compilado póstumamente en los tomos XIII, XIV, XV de la Husserliana (1973a; 1973b; 1973c). Así mismo, es necesario tener en cuenta, los manuscritos husserlianos sobre el mundo-de-la-vida sociocultural incluidos en Husserliana IV, XXIX y XXXIX (1997; 1993; 2008).

Siguiendo a Rizo-Patrón (2010), pueden distinguirse tres estratos de la intersubjetividad husserliana: el prerreflexivo o impulsivo, el reflexivo o mundano, y el social. El primero refiere a la constitución pasiva, instintiva, afectiva y corporal de una intersubjetividad primaria. El segundo, a la constitución perceptiva del alter ego. Por último, Husserl, también analiza la co-constitución del mundo histórico-cultural por una intersubjetiva trascendental bilateralmente abierta. 